NCC2-374

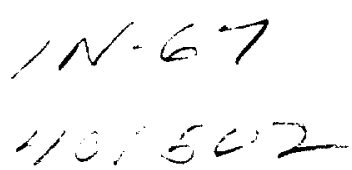

\title{
ON SUPERSTABILITY OF SEMIGROUPS
}

\author{
A. V. Balakrishnan \\ Flight Systems Research Center \\ UCLA
}

\begin{abstract}
July 1997
Presented at the 18th IFIP TC-7 Conference on System Modelling and Optimization, July 1997.

To be published in the Proceedings.
\end{abstract}




\title{
ON SUPERSTABILITY OF SEMIGROUPS
}

\author{
A. V. Balakrishnan \\ Flight Systems Research Center \\ UCLA
}

\begin{abstract}
This paper presents a brief report on superstable semigroups - abstract theory and some applications thereof. The notion of super stability is a strengthening of exponential stability and occurs in Timoshenko models of structures with self-straining material using pure (idealized) rate feedback. It is also relevant to the problem of Riesz bases of eigenfunctions of infinitesimal generators under perturbation.
\end{abstract}

\section{Introduction}

This paper presents a brief report on superstable semigroups and applications thereof. The notion of super stability introduced in [1] is a strengthening of exponential stability and occurs in Timoshenko models of structures with self-straining material using pure (idealized) rate feedback. It is also relevant to the problem of completeness of eigenfunctions of infinitesimal generators under perturbation. We begin with definitions and the associated abstract theory in Section 2. A general technique of constructing superstable semigroups is given in Sections 3. Section 4 deals with applications to "smart structure" theory. The relation to the preservation of the Riesz basis property of eigenfunctions of infinitesimal generators of semigroups under perturbation is outlined in Section 5.

\section{Definition and Basic Properties}

By a "semigroup," we shall mean a $C_{0}$-semigroup over a Hilbert space with compact resolvent, unless otherwise specified. Let $S(t), t \geq 0$ denote the semigroup and $\mathcal{H}$ the Hilbert space. Such a semigroup is said to be exponentially stable if

$$
\|S(t)\| \leq M \exp -\sigma t, \quad t \geq 0
$$


for some $\sigma, M>0$. The question: "Is there any stronger kind of stability?" has an affirmative answer - at least in infinite-dimensional theory. Thus the semigroup is said to be "superstable" (a notion introduced in [1]) if for every $\sigma>0, \exists M_{\sigma}$ such that

$$
\|S(t)\| \leq M_{\sigma} \exp -\sigma t, \quad t \geq 0 .
$$

The resolvent of a superstable semigroup is defined for every complex number $\lambda$ by:

$$
R(\lambda, A) x=\int_{0}^{\infty} e^{-\lambda t} S(t) x d t, \quad x \in \mathcal{H}
$$

and is thus an entire function - equivalently, the spectrum of $A$ is empty. In particular we see that $\mathcal{H}$ cannot be finite-dimensional - superstability is a truly infinitedimensional phenomenon - at least for linear systems.

Superstability is equivalent to saying that the stability index $\omega_{0}$ :

$$
\omega_{0}=\lim _{t \rightarrow \infty} \frac{\log \|S(t)\|}{t}=-\infty .
$$

In particular this is equivalent to saying that $S(t)$ must be quasi-nilpotent for every $t>0$ - the term superstable is used to emphasize the relation to problems of stability and stabilizability.

For the semigroup to be superstable it is necessary that the infinitesimal generator have an empty spectrum or, equivalently, the resolvent is an entire function. However the converse is not true; indeed one has only to construct a $C_{0}$ group whose generator has an empty spectrum - already described in the 1957 Hille-Phillips treatise $[2$, p. 667]; providing in fact a celebrated example for the failure of the spectral mapping theorem:

$$
\sigma[S(t)] \neq e^{t \sigma(A)} \quad \text { plus possibly zero }
$$

where $A$ denotes the infinitesimal generator. The point spectrum [2, p. 467] satisfies:

$$
P \sigma[S(t)]=e^{t P \sigma(A)} \quad \text { plus possibly zero. }
$$

It follows that a compact (or eventually compact) semigroup whose generator has an empty spectrum is superstable.

Since we are dealing only with a Hilbert space we should note that a complete characterization is given by the Greiner-Nagel ${ }^{\dagger}$ theorem $[3$, p. 96]: a semigroup is superstable if and only if

$$
\sup _{-\infty<\omega<\infty}\|R(\sigma+i \omega, A)\|<\infty
$$

for every $\sigma$ real, where $R(\lambda, A)$ denotes the resolvent of $A$. 
The resolvent of a superstable semigroup being an entire function, an immediate question is whether it is of finite order or not. We have in this connection a crucial result due to A. Sinclair [4].

Theorem (Sinclair) ${ }^{\dagger}$ Suppose the resolvent $R(\lambda, A)$ is an entire function of exponential type. Then the semigroup is superstable - actually nilpotent. In fact if

$$
\|R(\lambda, A)\| \leq M e^{|\lambda| T}
$$

then

$$
S(t)=0 \text { for } t>T \text {. }
$$

(and conversely, the converse being trivial).

Proof. The proof relies on a celebrated Paley-Wiener theorem on Fourier transforms, and an outline may be of interest to be included here.

Pick $\sigma>\omega_{0}$ and define

$$
r(z)=R(i z+\sigma, A)
$$

so that

$$
\|r(z)\| \leq c e^{|z| T}
$$

For $x, y$ in $\mathcal{H}$ and $t$ real:

$$
[r(t) x, y]=\int_{0}^{\infty} e^{-i t \xi} e^{-\sigma \xi}[S(\xi) x, y] d \xi
$$

and $[r(z) x, y]$ is an entire function such that

$$
\begin{gathered}
\| r(z) x, y] \mid \leq\|x\|\|y\| c e^{|z| T} \\
\int_{-\infty}^{\infty}\|r(t) x, y\|^{2} d t<\infty
\end{gathered}
$$

We can therefore apply the Paley-Wiener theorem (see $[5$, p. 375]) and obtain

$$
\begin{aligned}
{[r(z) x, y] } & =\int_{-T}^{T} F(\xi) e^{-i \xi z} d \xi \\
& =\int_{0}^{\infty} e^{-i z \xi} e^{-\sigma \xi}[S(\xi) x, y] d \xi
\end{aligned}
$$

Hence

$$
[S(\xi) x, y]=0 \text { for } \xi>T
$$

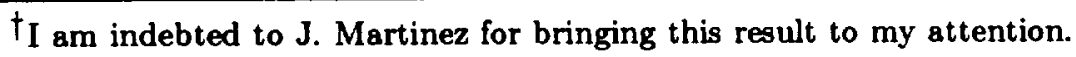


and hence, $x, y$ being arbitrary:

$$
S(t)=0 \text { for } t>T \text {. Q.E.D. }
$$

\section{Construction of Superstable Semigroups}

The simplest example of a superstable (actually nilpotent) semigroup is the shift semigroup on $L_{2}[0, L]$ for finite $L$. In $[2, \mathrm{p}$. 663, et seq.] Phillips uses RiemannLiouville fractional integration to construct a class of analytic superstable semigroups. We can generalize this construction considerably. Thus let $S(\cdot)$ denote a dissipative superstable semigroup. Define

$$
T(z) x=\frac{1}{\Gamma(z)} \int_{0}^{\infty} S(\xi) x \xi^{z-1} d \xi, \quad x \in \mathcal{H}, \operatorname{Re} z>0 .
$$

This is an analytic semigroup of class $H\left(\frac{-\pi}{2}, \frac{\pi}{2}\right)$ and is superstable along rays,

$$
z=r e^{i \theta}, \quad|\theta|<\frac{\pi}{2}
$$

since for any $\sigma>0$

$$
\|S(\xi)\| \leq M_{\sigma} \exp -\xi \sigma, \quad \xi \geq 0
$$

and consequently:

$$
\|T(z)\| \leq M_{\sigma} \int_{0}^{\infty} e^{-\sigma \xi}\left|\frac{\xi^{z-1}}{\Gamma(z)}\right| d \xi \leq M_{\sigma} \sigma^{-r \cos \theta} .
$$

Since

$$
T(z)=R(0, A)^{z},
$$

and $S(\cdot)$ is dissipative, so that $-R(0, A)$ is dissipative, we can invoke the theory of fractional powers of operators [6] for alternate expressions for (3.1) for $0<\operatorname{Re} z<1$. Thus we have:

$$
\begin{aligned}
T(z) x & =\frac{\sin \pi z}{\pi} \int_{0}^{\infty} \lambda^{z-1}(\lambda I+R(0, A))^{-1}(-R(0, A)) x d \lambda \\
& =\frac{-\sin \pi z}{\pi} \int_{0}^{\infty} \lambda^{-z} R(\lambda, A) x d \lambda \\
& =\frac{1}{\Gamma(-z)} \int_{0}^{\infty}\left(e^{-R(0, A) \xi}-I\right) x \xi^{-z-1} d \xi \\
& =\frac{1}{\Gamma(-z)} \int_{0}^{\infty}\left(e^{-R(0, A) \xi}-\sum_{0}^{n-1} \frac{(-R(0, A))^{k}}{k !}\right) \xi^{-z-1} d \xi, \\
& \text { for } n-1<\operatorname{Re} z<n
\end{aligned}
$$


from which we see in particular that $T(\cdot)$ is compact if $R(0, A)$ is. (The compactness of the resolvent of $A$ is irrelevant for the superstability property.)

Using Kato's formula (see [7, p. 260]) we have

$$
(\lambda I+T(z))^{-1}=\frac{\sin \pi z}{\pi} \int_{0}^{\infty}(r I+R(0, A))^{-1}\left[\frac{r^{z}}{\lambda^{2}-2 \lambda r \cos \pi z+r^{2 z}}\right] d r, 0<\operatorname{Re} z<1
$$

which is of course defined for every $\lambda \neq 0$. Contrast this with the representation using the Mittag-Leffler function in [2, p. 668].

Let $\mathcal{A}$ denote the generator of $T(\cdot)$. Then $\mathcal{A}$ has the representation for $x \in \mathcal{D}(A)$ :

$$
\mathcal{A} x=\gamma x-A \int_{0}^{\infty} S(\xi) x \log \xi d \xi
$$

where $\gamma$ is Euler's constant; and

$$
\mathcal{R}(\lambda, \mathcal{A}) x=\int_{0}^{\infty} S(\xi) x E(\lambda, \xi) d \xi
$$

where

$$
E(\lambda, \xi)=\int_{0}^{\infty} e^{-\lambda t} \xi^{t-1} \frac{1}{\Gamma(t)} d t
$$

from which, as already noted in $[2$, p. 667], we see that $\mathcal{R}(\lambda, \mathcal{A})$ is an entire function of infinite order (still satisfying (2.1)) so that in particular $T(z)$ is not nilpotent, for $\operatorname{Re} z>0$.

Remark. Phillips shows, based on some hard analysis results of Kober (see [2, p. 665]), that for the special case where $S(\cdot)$ is the left shift on $L_{2}[0,1]$, the "boundary values"

$$
\begin{aligned}
J(\eta) x & =\lim _{\sigma \rightarrow 0} T(\sigma+i \eta) x, \quad-\infty<\eta<\infty \\
& =-A T(1+i \eta) x
\end{aligned}
$$

determine a $C_{0}$ group whose generator $(i \mathcal{A})$ has an empty spectrum. But $J(\cdot)$ is of course not superstable.

Since

$$
R(\lambda, i \mathcal{A})=-i R(-i \lambda, \mathcal{A})
$$

we see that $R(\lambda, i \mathcal{A})$ is also an entire function of $\lambda$ of infinite order. In particular we see that the resolvent may be an entire function of infinite order and yet the semigroup need not be superstable. 


\section{Generalization: Hille-Phillips Calculus}

We can generalize the construction (3.1) using the Hille-Phillips operational calculus [2, Chapter 15]. For example let for each $t>0, a(t, \cdot)$ denote a set function on $[0, \infty)$ and such that (in the notation of $[2]$ ):

$$
\int_{0}^{\infty}\|S(\xi)\| d|a(t, \xi)|<\infty
$$

for which it is enough if

$$
\int_{0}^{\infty} e^{-\sigma_{0} \xi} d|a(t, \xi)|<\infty \text { for some } \sigma_{0}>0
$$

Let us assume this. Let

$$
\int_{0}^{\infty} e^{\lambda \xi} d a(t, \xi)=e^{t \phi(\lambda)}, \quad \operatorname{Re} \lambda \leq \sigma_{0}, t>0
$$

Then under suitable conditions on $a(t, \cdot)$ (equivalently $\phi(\cdot))$ :

$$
T(t) x=\int_{0}^{\infty} S(\xi) x d a(t, \xi)
$$

defines a $C_{0}$-semigroup, whose generator has an empty spectrum. The condition that

$$
\int_{-\infty}^{\infty}\left|e^{t \phi(\sigma+i \tau)}\right| d \tau<\infty, \quad t>0
$$

is sufficient for the semigroup to be superstable. This follows essentially from Lemma 16.3.1 of [2] which in turn is a consequence of the representation:

$$
T(t) x=\frac{1}{2 \pi i} \int_{\gamma-i \infty}^{\gamma+i \infty} e^{t \phi(\lambda)} R(\lambda, A) x d \lambda, \quad \operatorname{Re} \lambda \leq-\sigma_{0}
$$

For example we may consider fractional powers of $(-A)$. Define

$$
T(t) x=\int_{0}^{\infty} S(\xi) x F(t, \xi) d \xi, \quad t>0
$$

where $F(t, \cdot)$ is nonnegative and is defined by the Levy "stable" density:

$$
\int_{0}^{\infty} e^{\lambda \xi} F(t, \xi) d \xi=e^{-t(-\lambda)^{\alpha}}, \quad \operatorname{Re} \lambda<-\sigma_{0}, 0<\alpha<1
$$


The semigroup is superstable with generator

$$
-(-A)^{\alpha}
$$

The superstability can be proved directly from (3.9), since

$$
\|T(t)\| \leq M_{\sigma} e^{-t \sigma^{\alpha}} .
$$

Superstability holds in fact generally for $F(t, \cdot)$ nonnegative.

Let us also note that $\left(-\mathcal{A}^{2}\right)$ generates a $C_{0}$-semigroup, when $(i \mathcal{A})$ generates a $C_{0}$ group. Moreover using:

$$
\mathcal{R}\left(\lambda,-\mathcal{A}^{2}\right)=\frac{R(-i \sqrt{\lambda}, \mathcal{A})-R(i \sqrt{\lambda}, \mathcal{A})}{2 i \sqrt{\lambda}}
$$

we can readily verify that $(2.1)$ is satisfied and thence superstability. The semigroup generated has the representation

$$
T(t) x=\int_{-\infty}^{\infty} J(\xi) x G(\xi, t) d \xi, \quad t>0
$$

where $J(\cdot)$ denotes the group and

$$
G(\xi, t)=\frac{1}{2 \sqrt{\pi t}} \exp \frac{-\xi^{2}}{4 t}
$$

and is actually an analytic semigroup.

\section{Applications}

An early example in applications of superstability arising in the solution of a boundary value problem for partial dfferential equations - a "disappearing solution," the semigroup being actually nilpotent - is given by Majda [8]. More generally the phenomenon occurs in Timoshenko models $\ddagger$ of structures using self-straining material - "smart structures" - for feedback control $[9,10]$. The simplest such example is the "smart string" described by:

$$
\begin{gathered}
\rho \ddot{\theta}(t, s)-c \theta^{\prime \prime}(t, s)=0, \quad 0<s<\ell \\
-c \theta^{\prime}(t, 0)+g \dot{\theta}(t, 0)=0 ; \quad \theta(t, \ell)=0 ; c, \rho>0
\end{gathered}
$$

\footnotetext{
$\ddagger_{\text {It }}$ is curious that the phenomenon does not occur in Euler models.
} 
where the superdots indicate time derivatives and the primes the space derivatives, and $g>0$ is the variable gain parameter. The semigroup solution (see [9]; the semigroup is dissipative) as a function of $g$ becomes superstable at a critical value of $g$ given by

$$
g=\sqrt{\rho c} .
$$

The energy decays to zero in finite time and of course there are no modes. The solution vanishes ("disappears") for $t>2 \ell \sqrt{\rho / c}$ as can be directly verified (see [9]). A more realistic version [10] where we consider both displacement and torsion is given by:

$$
\begin{gathered}
m_{2} \ddot{v}(t, s)-c_{4}\left(v^{\prime \prime}(t, s)-\phi^{\prime}(t, s)\right)=0, \quad 0<s<\ell ; 0<t \\
m_{6} \ddot{\phi}(t, s)-c_{2} \phi^{\prime \prime}(t, s)-c_{4}\left(v^{\prime}(t, s)-\phi(t, s)\right)=0 \\
\phi(t, 0)=v(t, 0)=0 \\
c_{4}\left(v^{\prime}(t, \ell)-\phi(t, \ell)\right)+g \dot{v}(t, \ell)=0 \\
c_{2} \phi^{\prime}(t, \ell)+g \dot{\phi}(t, \ell)=0
\end{gathered}
$$

and $g$ is again the variable gain parameter. We have superstability for

$$
g=\sqrt{m_{6} c_{2}}=\sqrt{c_{4} m_{2}} .
$$

The resolvent can be shown to be of exponential type (details will appear elsewhere) and we have thus actually again a "disappearing solution."

\section{Relation to Bases}

We refer to a subspace as a superstable invariant subspace if it is an invariant subspace for the semigroup which is superstable thereon - in particular such a subspace cannot contain any eigenfunctions of the generator. The latter is the case if we consider the subspace orthogonal to the subspace spanned by the eigenfunctions of the adjoint of the generator. This relationship has already been noted by Majda [8], and can provide a technique for verifying whether Riesz bases are retained under a perturbation [1].

\section{Acknowledgement}

I am indebted to J. Martinez and P. Vu for many fruitful discussions and helpful comments.

Research support in part under NASA Grant No. NCC 2-374. 


\section{References}

1. A.V. Balakrishnan. "On Superstable Semigroups of Operators," Dynamic Systems and Applications, Vol. 5 (1996), pp. 371-384.

2. E. Hille and R.S. Phillips. Functional Analysis and Semigroups. AMS Colloquium Publications, Vol. 31. 1957.

3. R. Nagel, ed. One-parameter Semigroups of Positive Operators, Lecture Notes in Mathematics Series. Springer-Verlag, 1980.

4. A. Sinclair. Continuous Semigroups in Banach Algebras, London Mathematical Society Lecture Notes Series, No. 63. Cambridge University Press, 1982.

5. W. Rudin. Real and Complex Analysis, 3rd Edition. McGraw-Hill, 1987.

6. H.O. Fattorini. The Cauchy Problem. Addison-Wesley Publishing Co., 1983.

7. K. Yosida. Functional Analysis, 4th Edition. Springer-Verlag, 1974.

8. A. Majda. "Disappearing Solutions for the Dissipative Wave Equation," Indiana University Math. Journal (1975), pp. 1119-1133.

9. A.V. Balakrishnan. "Vibrating Systems with Singualr Mass-Inertia Matrices." In: First International Conference on: Nonlinear Problems in Aviation $\&$ Aerospace. Ed. S. Sivasundaram. Embry-Riddle Aeronautical University Press, 1996. Pp. 23-32.

10. A.V. Balakrishnan. "Smart Structures and Super Stability." In: Computational Science for the 21st Century. Ed. Jacques Periaux, et al. Chichester, England: John Wiley \& Sons, Ltd., 1997. 